\title{
The development and characterisation of high temperature fixed points at NMISA
}

\author{
N. Nel-Sakharova* \\ National Metrology Institute of South Africa (NMISA), Pretoria, South Africa
}

Received: 10 April 2015 / Accepted: 4 June 2015

\begin{abstract}
Many National Metrology Institutes (NMIs) realise the spectral irradiance scale by obtaining traceability from a cryogenic radiometer through the use of calibrated filter radiometers. The filter radiometers are used to determine the temperature of a high temperature black body which is then used as a reference source, which spectral radiance can be determined from Planck's equation. The uncertainty of the temperature measurement makes the most significant contribution to the uncertainty of realising the spectral irradiance scale. High temperature fixed points (HTFPs), above the copper point, can be used to improve these uncertainties. After more than ten years of research, results obtained on metal-carbon eutectic fixed points by several NMIs, showed that these novel high temperature fixed points could lead to significant improvements in high temperature metrology and could be considered as potential fixed points in a future International Temperature Scale. This paper describes the development and characterisation of selected high temperature metal-carbon fixed points at NMISA. It is demonstrated that these fixed points can be utilised as reproducible, stable reference standards for temperatures above the copper point.
\end{abstract}

Keywords: Eutectic cell, high temperature fixed point, phase transition, melt temperature, radiometry

\section{Introduction}

A wide range of industrial and medical applications require high precision spectral power distribution measurement of light sources, including solid state lighting, compact fluorescent lamps, conventional lamps, flat panel displays and solar radiation. The accuracy of these measurements is demonstrated by calibrating industrial reference standards against the national measurement standards for spectral irradiance, which are maintained by the National Metrology Institute of South Africa (NMISA).

Many National Metrology Institutes (NMIs) realise the spectral irradiance scale by obtaining traceability from a cryogenic radiometer through the use of calibrated filter radiometers. The filter radiometers are used to determine the temperature of a high temperature black body which is then used as a reference source, which spectral radiance can be determined from Planck's equation.

The uncertainty of the temperature measurement makes the most significant contribution to the uncertainty of realising the spectral irradiance scale. High temperature fixed points (HTFP), above the copper point, can be used to improve these uncertainties. After more than ten years of research, results obtained on metal-carbon eutectic fixed points by several NMIs, showed that these novel high temperature fixed points could lead to significant improvements in high temperature metrology and could be

* Correspondence: nnelsakharova@nmisa.org considered as potential fixed points in a future International Temperature Scale.

In 1996, the joint working group of the Comité Consultatif de Photométrie et Radiométrie CCPR) and the Comité Consultatif de Thermométrie (CCT) encouraged NMIs to develop high temperature fixed points above $2300 \mathrm{~K}$ with a reproducibility better than $100 \mathrm{mK}$ [1]. Three years later, in 1999, Yamada et al. published the first results of melt and freeze of metal-carbon eutectic points [2]. Since then, many NMIs have initiated cooperative projects and comparisons to characterise these points in order to reach a consensus on the transition temperatures and implementation procedures [3,4].

Some metal-carbon eutectic points (such as Co-C, $\mathrm{Pd}-\mathrm{C}, \mathrm{Pt}-\mathrm{C}$ and $\mathrm{Re}-\mathrm{C}$ ) are reaching such a level of confidence and are proving to be sufficiently reproducible, through comparisons and long-term studies [5]. Other fixed points, such as the metal carbide-carbon (MC-C) fixed points for radiometry applications, still require further study before temperatures can be assigned to them with acceptable uncertainties.

The NMISA constructed and characterised two Re-C eutectic cells to investigate its suitability as high temperature reference standards. Re-C was selected as it is the metal-carbon eutectics with the highest melt temperature, allowing interpolation from the copper point over the full temperature range required for NMISA applications. This eutectic has also been extensively studied internationally 


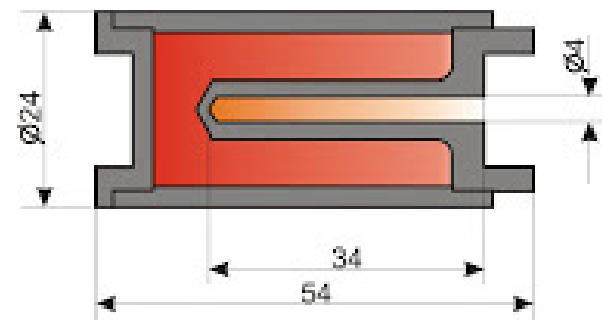

Fig. 1. Crucible dimensions (in $\mathrm{mm}$ ).

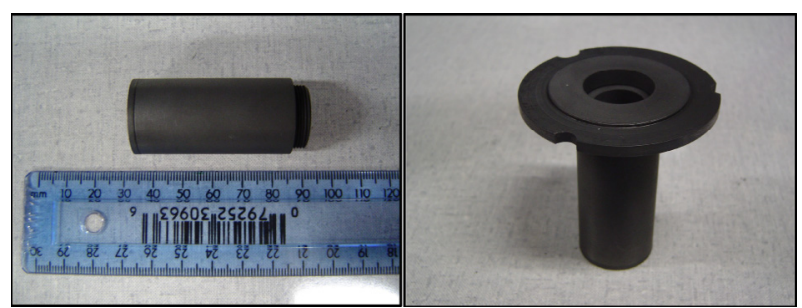

Fig. 2. Graphite crucible (left) fitted with a ring-holder (right).

and therefore provides a suitable reference to compare results with.

Provisional melt temperature values for a limited subset of eutectics have been published and the recommended melt temperature for Re-C is $2474.2{ }^{\circ} \mathrm{C} \pm 1.0{ }^{\circ} \mathrm{C}$ (which is equal to $2747.4 \mathrm{~K} \pm 1.0 \mathrm{~K}(k=2))$ [5].

This paper presents the results of development work and measurements at the NMISA and compares the results obtained here with those of other NMI.

\section{Experimental setup}

\subsection{Eutectic cell preparation}

Graphite crucibles supplied by the All-Russian Research Intitute of Optical and Physical Measurements (VNIIOFI) and designed to fit into the high-temperature black body BB3200pg (used as a furnace), were used. The cells were made from fine-grained pure $(>99.9995 \%)$ graphite. The construction was similar to the eutectic-containing crucibles commonly used by national metrology institutes (NMIs) and was cylindrical, with a length of $54 \mathrm{~mm}$ and a diameter of $24 \mathrm{~mm}$. The black body cavity in the centre of the cylindrical crucible had a depth of $35 \mathrm{~mm}$, a diameter of $4 \mathrm{~mm}$ and was conical at the bottom. The emissivity of the cavity was considered to be $\varepsilon=0.9995$. The crucible was fitted with a plug at the opposite end of the cavity opening to enable filling with the metal-carbon mixture. A diagram showing the crucible dimensions is given in Figure 1. Pictures of the crucibles used are shown in Figure 2.

Pure carbon (99.9999\%) and metal powders (99.999\%) were used to prepare the eutectic mixture. Table 1 shows the material purities and mixture proportions for each type of eutectic. The metal and graphite powders were supplied by the company Alfa Aesar. The purities shown
Table 1. Material specifications and powder mixing proportions.

\begin{tabular}{ccc}
\hline Material & Purity & $\begin{array}{c}\text { Proportion of graphite } \\
\text { in mixture (per weight) }\end{array}$ \\
\hline Rhenium (Re) & $99.999 \%(5 \mathrm{~N})$ & $1.0-1.5 \%$ \\
Graphite powder & $99.9999 \%(6 \mathrm{~N})$ & $\mathrm{n} / \mathrm{a}$ \\
$\begin{array}{c}\text { Solid graphite } \\
\text { (crucible material) }\end{array}$ & $99.9995 \%(5 \mathrm{~N} 5)$ & $\mathrm{n} / \mathrm{a}$ \\
\hline
\end{tabular}

in the table were provided by the supplier. The powders were mixed in an air-tight "glove-box" in an argon atmosphere.

The empty crucible and black body were purified by annealing above the eutectic material melting temperature (at approximately $2800 \mathrm{~K}$ ) for $1 \mathrm{~h}$. It was then filled with a metal-carbon powder mixture in an argon atmosphere and placed inside the black body (in the centre of the main cavity with the plug facing the opening) operated in a vertical position. The black body cavity was purged with argon to prevent electrical arcing while heated to melt the powder mixture to form the eutectic alloy. The filling process involved heating the crucible with the powder mixture to approximately $20 \mathrm{~K}$ above the eutectic melt point, cooling to room-temperature, re-filling with the powder mixture and re-heating to approximately $20 \mathrm{~K}$ above the melt point. This process was repeated until the crucible was completely filled with the eutectic alloy. About twenty (typical for Re-C) mixture-adding and melting cycles were necessary to fill each crucible completely.

\subsection{Measurement of melt temperature}

A linear pyrometer (LP4), calibrated in terms of ITS-90 by the Physikalisch-Technische Bundesanstalt (PTB) in Germany, was used to measure the phase transition temperatures of the Re-C cells. It was operated with an interference filter with a nominal centre wavelength of $650 \mathrm{~nm}$ and a bandwidth of approximately $20 \mathrm{~nm}$. The LP4 was focussed on the eutectic cell aperture plane (with the black body at room temperature) at a target distance of $750 \mathrm{~mm}$. The target size at this distance is $<1 \mathrm{~mm}$, which is significantly smaller than the cavity aperture, therefore the size-of-source effect should be negligible [6].

In order to measure the phase transition characteristics of the eutectic cells, the black body was operated in a horizontal position. The crucible was placed inside the black body cavity, at the centre, with the crucible cavity facing the LP4. This configuration is shown in Figure 3.

The black body was heated to approximately $20 \mathrm{~K}$ below the melt temperature of the eutectic material. Once the system had stabilised at this temperature, the current to the black body was increased and the eutectic melt was observed as a plateau in the pyrometer's signal versus time diagram. The melt finishes with a break-off point followed by a sharp temperature rise from the plateau to the set temperature of the black body. The system was again stabilised at approximately $20 \mathrm{~K}$ above the melt temperature. Thereafter, the current to the black body was decreased 


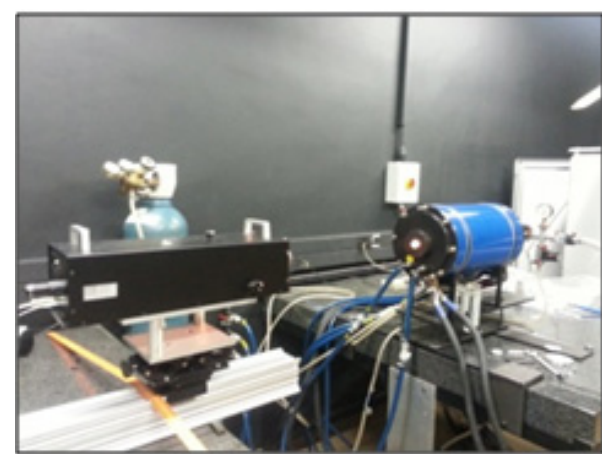

Fig. 3. Measurement configuration.

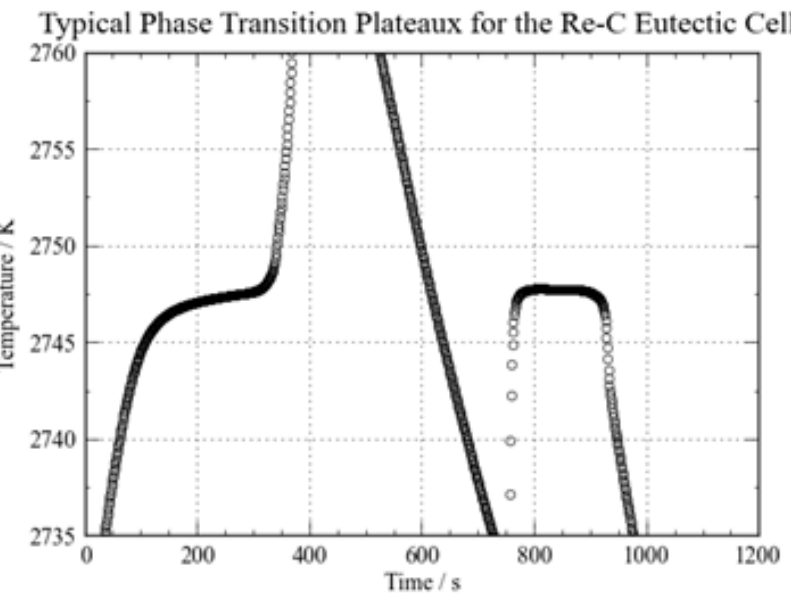

Fig. 4. Typical melt and freeze plateaux for the Re-C eutectic cells.

to reach a temperature below the freezing point of the eutectic material. The freeze plateau was observed, usually after undergoing super cool. The temperature of the system was stabilised at approximately $20 \mathrm{~K}$ below the freeze temperature before the next cycle was started.

The unique temperature at which the eutectic material melts is the liquidus point. It is defined as the maximum temperature at which crystals can be present in the melt at thermal equilibrium (i.e. it is the temperature at which the last solid melts) [7]. In practice, the presence of impurities in the eutectic material obscures the liquidus point. The point of inflection (POI) of the melting curve has been found to be a reproducible indicator of the melt temperature [8] and is being used in most studies.

\section{Results and discussions}

The phase transition temperatures of two different Re-C eutectic cells were measured (denoted Re-C (1) and Re-C (2), respectively). Over 3 days altogether, 13 melt-andfreeze cycles of the Re-C (1) cell were measured, while 14 cycles of the ReC (2) cell was measured over 4 different days. A typical melt-and-freeze cycle for Re-C eutectic cells is shown in Figure 4. A temperature plateau indicates a phase transition.

A melting plateau observed for the $\mathrm{ReC}$ (1) cell is shown in Figure 5. The first derivative of melting tem-

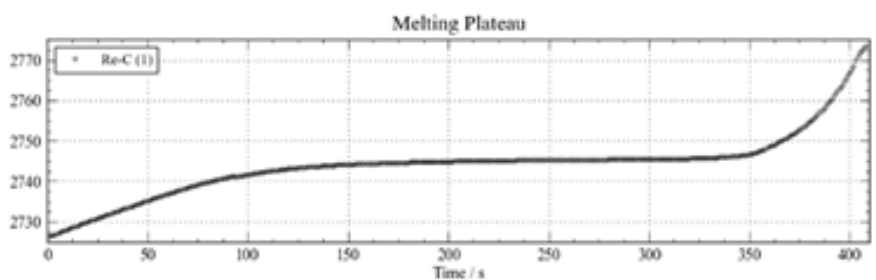

Fig. 5. A typical melting plateau observed for Re-C eutectic cells.

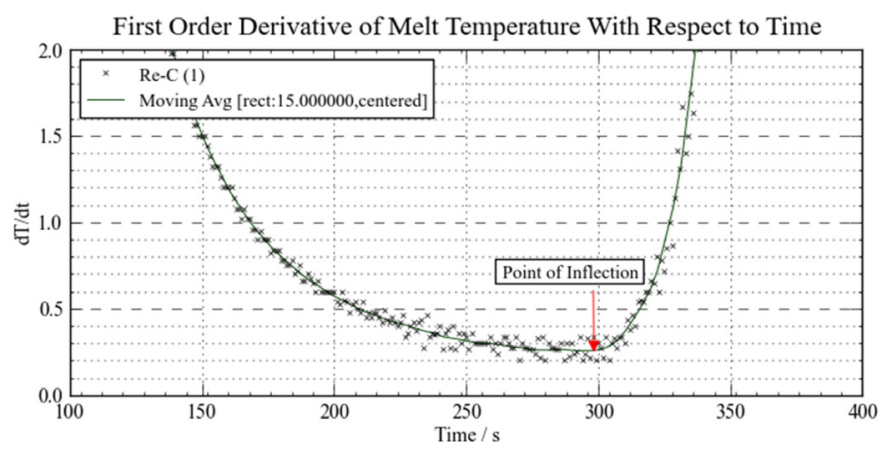

Fig. 6. Determination of the point of inflection of the melting plateau for a Re-C eutectic cell.

perature with respect to time is shown in Figure 6. The melt temperature is defined as the minimum of this curve (i.e. the POI) and has been estimated after applying a moving average curve smoothing technique.

At first, measurements were performed on the Re-C (2) eutectic cell. In order to utilise the existing measurement configuration, the LP4 was initially (over day 1 and 2) focussed on the eutectic cell aperture plane at a distance of $1 \mathrm{~m}$, with a cylindrical eutectic holder which did not allow convenient alignment. It was thus decided to adapt the measurement setup to specifically accommodate the measurement of eutectics. The distance between the LP4 and eutectic cell was changed to $750 \mathrm{~mm}$ and the cylindrical crucible holder was replaced with a ring-holder (shown in Fig. 2). All subsequent measurements were performed in this measurement configuration.

The measurement results for all melt-and-freeze cycles per day for each eutectic cell, are presented graphically in Figures 6 and 7 .

A difference of $277 \mathrm{mK}$ is observed between the average melt temperatures measured on days 1 and 2, versus days 3 and 4, for the Re-C (2) eutectic cell. Since this difference is significantly larger than the standard deviation for all subesequent measurements $(\leqslant 61 \mathrm{mK})$, it is attributed to the change in focal distance of the LP4 as well as improvement of the ease of alignment.

The average melt temperatures (in kelvin) measured on each day for the Re-C (1) and Re-C (2) cells are given in Tables 2 and 3, respectively.

The average melt temperature for both the Re-C (1) and $\mathrm{ReC}(2)$ cells is $2747.5 \mathrm{~K}$ with a measurement uncertainty of of $2.5 \mathrm{~K}(k=2)$. The reproducibility of the melt temperature was found to be within $100 \mathrm{mK}$. 


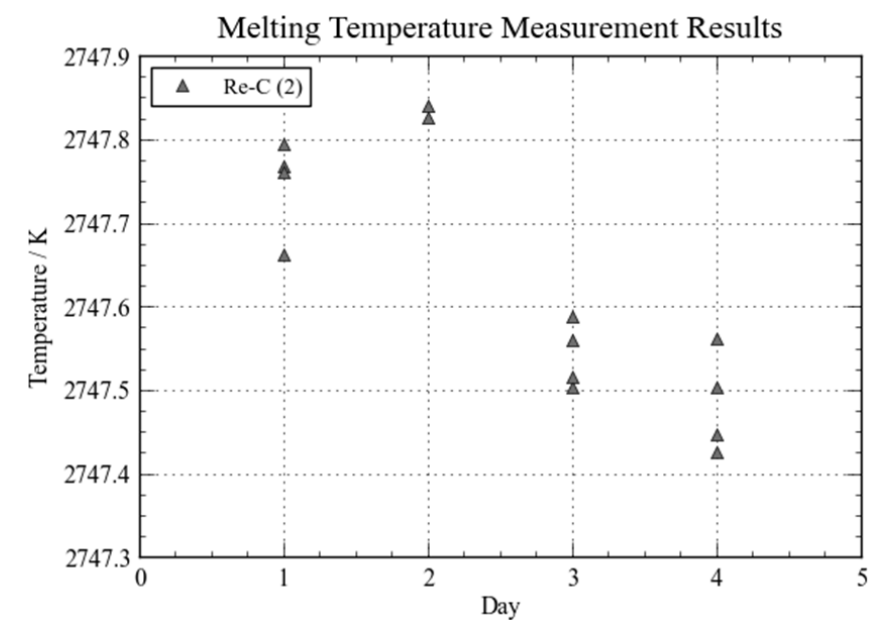

Fig. 7. Melt temperatures of the Re-C (2) cell measured over four different days.

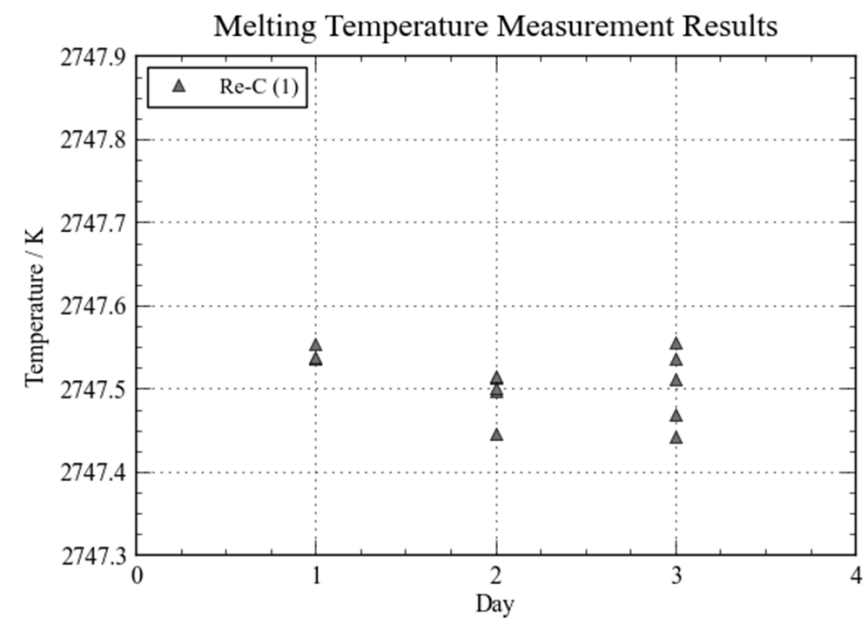

Fig. 8. Melt temperatures of the Re-C (1) cell measured over three different days.

Table 2. Melt temperatures of the Re-C (1) eutectic cell measured with the LP4 (in kelvin).

\begin{tabular}{cccc}
\hline Re-C (1) & Day 1 & Day 2 & Day 3 \\
No. of melt/freeze cycles & 3 & 5 & 5 \\
\hline Average & 2747.54 & 2747.49 & 2747.50 \\
Standard deviation & 0.01 & 0.03 & 0.05 \\
\hline
\end{tabular}

\section{Conclusion}

The results presented in this paper show that the melt temperature of the ReC eutectic cells constructed and characterised by the NMISA is in agreement with the internationally published values within the stated uncertainties.
Table 3. Melt temperatures of the Re-C (2) eutectic cell measured with the LP4 (in kelvin).

\begin{tabular}{ccc}
\hline Re-C (2) & Day 3 & Day 4 \\
No. of melt/freeze cycles & 4 & 4 \\
\hline Average & 2747.54 & 2747.48 \\
Standard deviation & 0.04 & 0.06 \\
\hline
\end{tabular}

The important outcome is that the repeatability of these Re-C cells is sufficient to be utilised as stable reference standards for temperatures above the copper point. This provides the NMISA with a practical means to verify the stability of its radiometric (spectral radiance and spectral irradiance) and temperature scales.

\section{References}

1. Comité Consultatif de Thermométrie, Rapport de la 19e Session, Bureau International des Poids et Mesures, Paris (1998)

2. Y. Yamada, H. Sakate, F. Sakuma, A. Ono, Radiometric observation of melting and freezing plateaus for a series of metal-carbon eutectic points in the range $1330{ }^{\circ} \mathrm{C}$ to $1950{ }^{\circ} \mathrm{C}$, Metrologia 36, 207 (1999)

3. G. Machin, Y. Yamada, D. Lowe, N. Sasajima, K. Anhalt, J. Hartmann, R. Goebel, H.C. McEvoy, P. Bloembergen, A comparison of high temperature fixed-points of $\mathrm{Pt}-\mathrm{C}$ and Re-C constructed by BIPM, NMIJ and NPL, in Proc. of 9th Int. Symp. on Temperature and Thermal Measurements in Industry and Science (Tempmeko), Zagreb, Croatia, edited by Davor Zvizdic (LPM/FSB, 2005) p. 1049-56

4. K. Anhalt, J. Hartmann, D. Lowe, G. Machin, M. Sadli, Y. Yamada, Thermodynamic temperature determinations of Co-C, Pd-C, Pt-C and Ru-C eutectic fixed-point cells, Metrologia 43, S78 (2006)

5. M. Sadli, J. Fischer, Y. Yamada, V. Sapritsky, D. Lowe, G. Machin, Review of metal-carbon eutectic temperatures proposal for new ITS-90 secondary points, in Proc. of 9th Int. Symp. on Temperature and Thermal Measurements in Industry and Science (Tempmeko), Zagreb, Croatia, edited by Davor Zvizdic (LPM/FSB, 2005) p. 341-348

6. H.W. Yoon, D.W. Allen, R.D. Saunders, Methods to reduce the size-of-source effect in radiometers, Metrologia 42, 89 (2005)

7. D. Lowe, G. Machin, Evaluation of methods for characterising the melting curves of a high temperature cobalt-carbon fixed point to define and determine its melting temperature, Metrologia 49, 189 (2012)

8. E.R. Woolliams, G. Machin, D.H. Lowe, R. Winkler, Metal (carbide)-carbon eutectics for thermometry and radiometry: a review of the first seven years, Metrologia 43, R11 (2006) 